\title{
Computational Study of Uniaxial Deformations in Silica Aerogel Using a Coarse-Grained Model
}

\author{
Carlos A. Ferreiro-Rangel and Lev D. Gelb* \\ Department of Materials Science and Engineering,Richardson, TX 75080 \\ E-mail: lev.gelb@utdallas.edu
}

${ }^{*}$ To whom correspondence should be addressed 


\begin{abstract}
Simulations of a flexible coarse-grained model are used to study silica aerogels. This model, introduced in a previous study (J. Phys. Chem. C 111 [2007] 15792), consists of spherical particles which interact through weak nonbonded forces and strong interparticle bonds that may form and break during the simulations. Small-deformation simulations are used to determine the elastic moduli of a wide range of material models, and large-deformation simulations are used to probe structural evolution and plastic deformation. Uniaxial deformation at constant transverse pressure is simulated using two methods: a hybrid Monte Carlo approach combining molecular dynamics for the motion of individual particles and stochastic moves for transverse stress equilibration; and isothermal molecular dynamics simulations at fixed Poisson ratio. Reasonable agreement on elastic moduli is obtained except at very low densities. The model aerogels exhibit Poisson ratios between 0.17 and 0.24 , with higher-density gels clustered around 0.20 , and Young's moduli that vary with aerogel density according to a power-law dependence with exponent near 3.0. These results are in agreement with reported experimental values. The models are shown to satisfy the expected homogeneous isotropic linear-elastic relationship between bulk and Young's moduli at higher densities, but there are systematic deviations at the lowest densities. Simulations of large compressive and tensile strains indicate that these materials display a ductileto-brittle transition as the density is increased, and that the tensile strength varies according to a power law with density, with exponent in reasonable agreement with experiment. Auxetic behavior is observed at large tensile strains in some models. Finally, at maximum tensile stress very few broken bonds are found in the materials, in accord with the theory that only a small fraction of the material structure is actually load-bearing.
\end{abstract}

Keywords: aerogel, molecular simulations, coarse-grained modeling, porous materials, soft matter, statistical mechanics, elastic constants 


\section{Introduction}

Aerogels are amorphous porous materials which exhibit a unique combination of properties including high surface area, high porosity and low thermal conductivity. ${ }^{1}$ They are attractive for applications in adsorption, catalysis, thermal insulation, aerospace, energy, sensing, lighting, and other areas. ${ }^{2}$ Aerogels of many different compositions have been prepared, ranging from the original silica aerogels to other ceramics, pure carbon aerogels, organic/inorganic composites, biopolymer/silica composites, and aerogels supporting metallic nanoparticles, among other systems. Their structural and mechanical properties have been studied by many means, both experimental and computational; the current status of aerogel research is thoroughly reviewed in the recent Aerogel Handbook ${ }^{3}$.

Silica aerogels are the most intensively studied aerogels and so far the only ones used commercially on large scales. To prepare silica aerogel, a precursor species such as tetramethoxysilane (TMOS) or tetraethoxysilane (TEOS) is hydrolyzed to form Si-OH (silanol) groups, which then react to form Si-O-Si bridging bonds. ${ }^{4}$ Under suitable conditions this process leads rapidly to a solution ("sol") of small dense silica particles. ${ }^{5,6}$ Bond formation between these particles leads to small aggregates, which further agglomerate to form a gel, a mechanically rigid, system-spanning network. This "wet" gel may shrink and expel solvent through syneresis, which involves the formation of additional bonds. ${ }^{4}$ Finally, the wet gel is dried under conditions which prevent fracture due to capillary forces. ${ }^{5,6}$ The resulting materials have high porosity and surface area. They also have very low thermal conductivity, due to low connectivity and the presence of "dead ends" in the gel network. ${ }^{2}$ Although silica aerogels have been prepared with densities as low as $0.003 \mathrm{~g} / \mathrm{cm}^{3}$ (corresponding to a volume fraction of approximately 0.001), most experimental studies and proposed applications have focused on the density range $0.1-0.25 \mathrm{~g} / \mathrm{cm}^{3}$, corresponding to volume fractions of $0.05-0.12^{3}$.

The same structural characteristics that make aerogels interesting also make them brittle, hindering their practical application. The mechanical properties of aerogels have therefore 
been studied in some detail. Aerogels are elastic under small strain, but at larger strains undergo plastic deformation and irreversible structural changes. ${ }^{7}$ There have been several studies of aerogel bulk modulus, ${ }^{7-12}$ which is found to depend on the density via a power law $K \propto \rho^{m}$ with exponent $m$ varying from $3.0^{7}$ to $3.7^{8}$.

The Young's modulus, $Y$, which is the relevant elastic constant for uniaxial deformation, has been studied rather more intensively, using ultrasonic measurements ${ }^{10,13,14}$, three-point beam-bending ${ }^{8,15,16}$, uniaxial compression and tension ${ }^{17,18}$, and nanoindentation measurements ${ }^{11,19,20}$. All of these studies report power-law dependence of $Y$ on aerogel density, with exponents ranging from $3.0^{20}$ to $3.6^{13}$ to $3.8^{15}$ to $4.18,{ }^{17}$ with differences appearing to come from sample properties rather than experimental technique.

Direct measurements of the Poisson ratio $(\nu)$ have also been made. Gross et $a l .{ }^{21}$ reports values between 0.205 and 0.230 , almost independent of gel density. Gross and Scherer ${ }^{16}$ find $\nu=0.2$ in beam-bending experiments on a single sample. Pollanen et al. ${ }^{22}$ find $\nu=0.3 \pm 0.05$ from analysis of optical images, and Bhupathi et al. ${ }^{23}$ obtained $\nu$ between 0.094 and 0.143 for high-porosity samples through direct mechanical compression. Álvarez-Arenas et al. ${ }^{10}$ found $\nu=0.2$ from analysis of ultrasonic resonances of air-surrounded aerogel plates. We note that many experimental determinations of $Y$ simply assume $\nu=0.2^{7,9,24-26}$ or similar values, ${ }^{14,27}$ the same value as for dense vitreous silica.

Other mechanical properties also display power-law dependence with density. Woignier and Phalippou ${ }^{8}$ report a fracture strength exponent of 2.6 \pm 0.2 . Moner-Girona et $a l .{ }^{20}$ find that hardness varies according to an exponent of 2.0. Wong et al. ${ }^{18}$ report a tensile strength exponent of 2.3 for aerogels prepared from polyethoxydisiloxanes. They also report that samples with density above $0.2 \mathrm{~g} / \mathrm{cm}^{3}$ are brittle, while those with density below $0.1 \mathrm{~g} / \mathrm{cm}^{3}$ are ductile and very compressible.

Characterizations of aerogel are not always consistent, even when performed on the same sample. Gross et al. ${ }^{21}$ used sound velocity measurements and static compression experiments to obtain $Y$ and found that values from the two techniques disagreed at both the lowest and 
highest aerogel densities. The highest density aerogel appeared "softer" and the lowest density aerogel "harder" in the static experiment, a difference that remains unexplained. Stark et al. ${ }^{19}$ used AFM to study the elastic properties of commercial aerogel powder particles (Basogel, BASF) for which $Y \simeq 6 \mathrm{MPa}$, finding that the modulus varied somewhat with the position of the indenter on the aerogel.

Silica aerogels can be modified by physical or chemical processing to exhibit enhanced properties. Phalippou et al. ${ }^{28}$ showed that sintering improves the mechanical properties of aerogel and that $Y \propto \rho^{m}$ with $m \approx 3.3$. Miner et al. ${ }^{29}$ showed that the Young's modulus of hygroscopic aerogels increases with relative humidity. Rosa-Fox et $a l .{ }^{30}$ used nanoindentation to show that in hybrid organic/inorganic aerogels the modulus decreases with polymer content, while Meador et al. ${ }^{31}$ showed that the Young's modulus of silica aerogels is increased by isocyanate cross-linking. Additional strategies used to improve mechanical properties include functionalization with trimethoxymethylsilane ${ }^{32}$ and chemical vapor deposition (CVD) treatment with hexamethyldisilazane or hexachlorodisilane. ${ }^{33}$

Computer simulations based on atomistic, coarse-grained, and continuum methods have also been used to investigate the mechanical properties of aerogels. Most prior work in this area has focused on the bulk modulus, however, ${ }^{34-38}$ and only a few studies have addressed uniaxial deformations. Ma et al. ${ }^{39}$ applied a finite element method to measure elastic moduli in aerogel models prepared by means of an off-lattice DLCA simulation with dangling bond deflection, treating the gel as a network of linearly elastic beams. This calculation predicted a power-law dependence of $Y$ on density with exponent 3.6. They also report an increase in the Poisson ratio from 0.125 to 0.145 as the aerogel volume fraction increases from 0.03 to 0.18 (i.e., a porosity decrease from $97 \%$ to $82 \%$ ), and that beam bending is the main form of deformation when a strain is applied to the gel network. Barbero and Campo ${ }^{40}$ applied a stochastic coarse-grained approach to generate aerogel models and then calculated mechanical properties using a recursive multiscale scheme incorporating the finite element method. They found the expected power-law dependence of Young's modulus on density, 
and also that the mass distribution and connectivity of the gel structure had a large impact on both $\nu$ and $Y$.

We have previously introduced a coarse-grained model for studying aerogels which incorporates realistic interparticle bonds, stochastic chemical dynamics, Langevin-type diffusive motion, and thermal fluctuations. ${ }^{38,41}$ This model naturally accounts for important processes such as structural relaxation, bond breaking, and thermal motion during aerogel synthesis and mechanical deformation simulations. Our aerogel models are prepared by simulating the sol-gel process and then relaxing the structures obtained in a multi-stage procedure. The resulting models can then be structurally and mechanically characterized. The first study performed using this approach focused on the dependence of aerogel properties on the conditions imposed during gelation ${ }^{41}$. In particular, structural properties such as fractal dimension and mean pore size were shown to depend significantly on both the chemical reactivity of the sol and on the solvent viscosity, with higher viscosity and higher reactivity both leading to finer-textured gels with lower fractal dimension and mean pore size. This study considered models comprised of approximately 5000 sol particles, at volume fraction 0.05 (95\% porosity.) Although bulk moduli were measured, large uncertainties due to the relatively small system size meant that the influence of gel reactivity on this quantity could not be reliably identified. A subsequent study ${ }^{38}$ focused on more reliable determination of bulk moduli, using an improved post-gelation relaxation protocol, larger systems consisting of 12000 particles, and averaging of all data over three independent "realizations" (that is, model aerogels generated under the same conditions but starting from different initial configurations) in order to increase statistical quality and estimate uncertainties. With these parameters bulk moduli could be obtained reliably, with estimated uncertainties ranging between $20 \%$ for the lowest-modulus materials to $2 \%$ for the highest. The lowest-density materials are the softest, but also display the largest characteristic length-scales (such as mean pore size) and longest characteristics time-scales. Measurements at low density are therefore most susceptible to low statistical quality both from finite run-length and sample- 
to-sample variation. Nonetheless, this study found that the model aerogels displayed a power-law dependence of bulk modulus on density with an exponent of around 3.1 over the entire density range studied (volume fractions ranging from approximately 0.03 to 1.1 ). This value is consistent with many experimental reports as reviewed above. Reasonable quantitative agreement with experimental moduli was also obtained. The dependence of modulus on sol particle size and polydispersity was also investigated, with the finding that the key correlate for the bulk modulus appears to be the volumetric density of interparticle bonds, rather than the material density, as found by comparing the properties of aerogel models of the same mean particle size but different size polydispersities.

In the present work we consider uniaxial deformations of both small and large compressive and tensile strains under quasi-equilibrium isothermal conditions. Protocols for the calculation of the Poisson ratio and Young's modulus are developed and are shown to give consistent results except for materials of very low density. Good agreement is found with experimental results at both qualitative and quantitative levels. Simulations at large strains, both compressive and tensile, are then used to probe the crossover from elastic to plastic behavior. We demonstrate measurement of the tensile strength, the strain at maximum tensile stress, and other quantities. The aerogel models are found to display a ductile-to-brittle transition as the density is increased, and in some cases exhibit auxetic behavior under large tensile strain.

\section{Models and computational methods}

The model used in this work consists of smooth, spherical "primary" (sol) particles with density equal to that of amorphous silicon dioxide, $2.2 \mathrm{~g} / \mathrm{cm}^{341}$. These interact through weak nonbonded forces and strong interparticle bonds. This is similar to some previous models for colloidal gels ${ }^{42-44}$ and polymer gels, ${ }^{45}$ but with a much stiffer and more complex bonding potential incorporating both angular and torsional terms. The non-bonded forces 
between particles are modeled with a two-body shifted-center Lennard-Jones potential. The stretching of interparticle bonds is described with a Morse potential, while bond bending and torsional displacements are modeled with terms similar those used in standard atomistic force fields. The functional forms and parameters used are detailed in the Supplementary Information, along with selected other technical details of the simulations.

During a simulation, bonds may form when non-bonded particles collide. The probability $P_{\text {bond }}$ of creating a bond in a given time-step $\delta t$ is related to the forward reaction rate constant

$k_{f}$ through $P_{\text {bond }}=k_{f} \delta t$ (for small $\left.P_{b o n d}\right)^{46}$. By construction, bonds are created at the equilibrium bond length (minimum energy configuration) in order to avoid any discontinuity of forces and torques at bond creation. Two particles can share only one bond, and each particle can have a maximum of six bonds. ${ }^{38}$

Bonds may also be broken, allowing for simulation of extreme deformations and material failure. The various terms in the potential energy are all constructed so as to approach zero rapidly with bond extension. At a chemically reasonable maximum distance the bond can thus be considered broken and removed, again without introducing discontinuities in the forces $^{41}$.

\subsection{Aerogel model preparation}

The aerogel models used here are taken from the previous study of bulk moduli; ${ }^{38}$ their preparation is described in detail in that work. To briefly summarize, a multi-stage protocol consisting of initialization, gelation, and subsequent relaxations is followed. Sol particles are first randomly inserted (with avoidance of overlap) in the simulation cell until a target density is reached, and a short simulation by Langevin dynamics is used to thermalize the system. Gelation is then simulated using Langevin dynamics with interparticle bonding enabled. These simulations are run until well after the gel point is reached, defined by the incorporation of all sol particles into a single, system-spanning cluster; this required times ranging between $250 \mathrm{~ns}$ and $2100 \mathrm{~ns}$, depending on the system density and particle size. The 
as-formed gels are under tension, so in a second stage they are relaxed under isothermalisobaric conditions at zero pressure, for times ranging between 825 ns and 1250 ns. Bonding may still occur in this stage, which mimics the syneresis and network reorganization that occur during gel aging in experimental work. To accelerate relaxation, in these simulations particles move ballistically (that is, the implicit solvent is removed.) A further isothermalisobaric relaxation of length between $792 \mathrm{~ns}$ and $2560 \mathrm{~ns}$ is then performed under conditions where no further bonds may form, corresponding to aging of the "dry" aerogel (experimentally, formation of silica bonds is catalyzed by the solvent ${ }^{4}$ ); the gel re-expands a much smaller amount in this phase. The final model, now in a quasi-equilibrium state at zero pressure, is suitable for mechanical analysis.

The aerogel models used here all consisted of $N=12000$ primary particles. Three types of model aerogels are considered, which differ in primary particle diameter. Type "A" models have primary particles with diameters of $1.50 \mathrm{~nm}$, type "B" have $1.75 \mathrm{~nm}$ diameter particles, and type "C" have $2.00 \mathrm{~nm}$ particles. For each type, materials generating at five different initial volume fractions $\alpha_{\text {gel }}$ were considered, with $0.02 \leq \alpha_{\text {gel }} \leq 0.10$. Note that the density in these models is exactly proportional to the volume fraction as reported, so the two terms may be used almost interchangeably. Finally, three independent model realizations were prepared for each combination of volume fraction and particle size, such that a total of 45 separate model aerogel realizations were simulated. The properties reported for each type and volume fraction are averages over the three realizations, while the reported uncertainties are the standard deviation over the three values, as in previous work. ${ }^{38}$

\subsection{Uniaxial deformation simulations}

Measurement of the Young's modulus and Poisson ratio, and, more generally, study of the behavior of a system under uniaxial tension or compression, requires simulation under conditions of either fixed length (strain) or fixed applied stress in a chosen "axial" direction, and constant zero stress in the transverse directions. Molecular dynamics simulations un- 
der conditions of inhomogeneous stress require analytical calculation of the stress tensor at each time step. ${ }^{47}$ The choice of interparticle bond model used here involves up to fourbody forces acting at the surfaces of the particles, which greatly complicates calculation of the stress tensor. As a result, we have used two alternative strategies to simulate uniaxial deformation.

The first of these is a form of hybrid Monte Carlo, or HMC. ${ }^{48-52}$ In HMC short molecular dynamics (MD) trajectories of the whole system are treated as moves to be accepted or rejected within a larger Monte Carlo simulation. Our approach here is to use the MD trajectories to move the gel particles within the simulation cell at constant volume, and then to use conventional Monte Carlo cell-deformation moves to enforce the condition of constant transverse stress.

In MD trajectory "moves," new rotational and translational velocities for all particles in the system are first sampled from a Gaussian distribution of width determined by the simulation temperature, $T=300 \mathrm{~K}$, and then the microcanonical equations of motion are integrated for several time-steps. The final state is accepted with probability $P_{a c c}=$ $\min \{1, \exp [-\beta \Delta E]\}$, with $\beta=1 / k_{B} T$ and $E$ the total (kinetic plus potential) energy. If a move is rejected the coordinates of the particles are restored to the initial configuration state prior to the MD trajectory. A leap-frog algorithm ${ }^{41,53}$ is used to ensure that the trajectories are time-reversible, as required for the algorithm to satisfy detailed balance. ${ }^{51}$ The fraction of trajectory moves accepted depends on both the time step of integration and the number of steps in the trajectory. We fixed the number of MD steps to 10 and chose the time step to give an acceptance ratio between 0.6 and 0.7 , since $P_{A}=0.651$ has been suggested as optimal for HMC simulations. ${ }^{52}$

Constant transverse stress is achieved through the use of random perturbations to the cell dimensions orthogonal to the strain. The material is assumed to be isotropic, so that the two tranverse cell dimensions are kept the same. If the strain is applied along direction $i$, then the trial volume change is $\Delta V=\left(2 r_{n}-1\right) \Delta A_{\max } L_{T}$, where $r_{n}$ is a uniform random 
variable on $[0,1]$ and $L_{T}$ is the length of the simulation cell in the transverse directions. Choosing a maximum change $\Delta A_{\max }=0.03 \%$ yields an acceptance ratio between 0.6 and 0.7 in these simulations. Area-change moves are accepted according to the probability, ${ }^{53}$

$$
P_{V, a c c}=\min \left\{1, \quad \Delta U+P_{e x t} \Delta V-N k_{B} T \ln \left(\frac{V+\Delta V}{V}\right)\right\}
$$

where $\Delta U$ is the associated change in potential energy, $N$ is the number of particles, $V$ is the initial volume, and $P_{\text {ext }}$ is the external transverse pressure, here zero (since these are highly porous materials, the effect of atmosphere is not included.)

\subsubsection{Measurement of mechanical properties}

The actual simulation protocol used for the measurement of $Y$ and $\nu$ is based on applying one cycle of a sinusoidally varying strain under quasi-equilibrium conditions, accomplished by making many small changes in one dimension of the simulation cell and following each with short equilibration and data-collection stages. In aerogel models with volume fractions $\alpha_{\text {gel }} \leq 0.03$, the simulation cell length along a chosen axis is varied sinusoidally over a $\pm 5 \%$ range in 8000 discrete steps, returning to its original value both at the midpoint and end of the

simulation. For aerogels with $\alpha_{\text {gel }}>0.03$ a similar procedure is used but applying strains of only $\pm 2.5 \%$, to avoid fracture due to the brittleness exhibited by these materials. In the lowdensity systems, the maximum discrete change in axial length is approximately $0.004 \%$, while in the high-density systems it is $0.002 \%$. After each such change, 1000 Monte Carlo moves are performed, consisting of either HMC trajectories as described above or perturbations to the transverse area, chosen with equal probability. Bond breakage is disabled during the first 650 moves, to ensure that the bond-breaking is a result of the tension undergone by the model and not due to the instantaneous particle motion due to change in cell dimensions. Data collection is performed only over the second half of the 1000-move cycle. The complete sinusoidal cycle is therefore spread over 8 million Monte Carlo moves; 4 million transverse- 
area moves and 4 million HMC moves, corresponding very roughly to an MD trajectory of at least 600 ns duration (assuming 60\% of moves accepted and 0.025 ps time-steps.) These conditions are sufficiently gentle to ensure that well below $0.1 \%$ of interparticle bonds are broken during the simulations. This fraction is small enough to have no significant effect on the resulting moduli, which was confirmed in selected cases by repeating the sinusoidal perturbation more than once.

During these simulations, after every 10th application of strain the isothermal stresses along the cell axes $\sigma_{i}$ are calculated by numerical differentiation of the total energy as a function of applied strain $\epsilon_{i}=\Delta L_{i} / L_{i}^{0},{ }^{54}$

$$
\sigma_{i}=\frac{1}{V_{0}} \frac{\delta E}{\delta \epsilon_{i}}=\frac{1}{V_{0}}\left[\left\langle\frac{\delta U}{\delta \epsilon_{i}}\right\rangle-k_{B} T N\right]
$$

Measurement of the axial stress $\sigma_{\text {axial }}$ as a function of strain $\epsilon_{\text {axial }}$ yields the isothermal stress-strain curve, from which we estimate the isothermal Young's modulus $Y=\sigma / \epsilon$. The Poisson ratio is obtained as the slope of the transverse strain vs. the axial strain curve $\nu=-d \epsilon_{\text {trans }} / d \epsilon_{\text {axial }}$, determined by linear regression in the elastic region. The transverse strain is obtained simply by measuring the average transverse cell dimensions at each axial strain.

As the HMC approach is untested for the model and material of interest here, we also consider an alternative simulation approach based on conventional molecular dynamics. In this method, we also simulate a sinusoidally-varying uniaxial strain, but now with a fixed Poisson ratio $\nu$ as input (which determines the tranverse strain.) The sinusoidal perturbation is applied over $3 \times 10^{6}$ time steps. The dynamics include the possibility of bond breakage but not of additional bond formation. The isothermal stress along each cell axis was calculated numerically every 1000 time-steps. The true $\nu$ is extracted from these simulations using an interpolation procedure described below. Further details concerning these simulations are given in the Supplementary Information. 


\subsection{Large deformation simulations}

For HMC-based determination of maximum tensile stresses and study of the evolution of aerogel structure under large deformations, slightly different protocols were used. For tensile simulations, a 20\% strain was applied linearly over 5000 discrete steps. Each step consisted of elongation of the simulation cell followed by a block of 2000 Monte Carlo moves (either HMC or transverse-area, as above), with data collection performed only over the latter 1000 moves of each block. For compressive strain simulations, a strain of $40 \%$ was applied over 5000 discrete steps, with relaxation and data collection as in the tensile strain simulations.

\section{Results and discussion}

\subsection{Elastic-range simulations and mechanical characterization}

Table 1: Mechanical properties of model aerogels. $d$ is the primary particle diameter, $\alpha_{g e l}$ is the volume fraction of the gels, $\rho_{a}$ is the gel density, and $Y_{H M C}$ and $Y_{M D}$ are the Young's modulus calculated by HMC simulations and by MD simulations (with $\nu=0.20$ ) respectively. $\nu_{H M C}$ is the Poisson ratio resulting from the HMC simulations, while $\nu_{M D}$ is the Poisson ratio estimated from MD simulations. $\epsilon_{x}^{\max }$ is the strain at which the maximum tensile strength $\sigma_{x}^{\max }$ is found, and $B_{b}^{\max }$ is the percentage of broken bonds at that point. Numbers in parentheses are uncertainties in the last digit, obtained by comparison of the three independent model realizations run for each system.

\begin{tabular}{ccccccccccc}
\hline Model & $\begin{array}{c}d \\
{[\mathrm{~nm}]}\end{array}$ & $\alpha_{g e l}$ & $\begin{array}{c}\rho_{a} \\
{\left[\mathrm{~g} / \mathrm{cm}^{3}\right]}\end{array}$ & $\begin{array}{c}Y_{H M C} \\
{[\mathrm{MPa}]}\end{array}$ & $\nu_{H M C}$ & $\begin{array}{c}Y_{M D} \\
{[\mathrm{MPa}]}\end{array}$ & $\nu_{M D}$ & $\begin{array}{c}\epsilon_{x}^{\max } \\
\sigma_{x}^{\max } \\
{[\mathrm{MPa}]}\end{array}$ & $\begin{array}{c}B_{b}^{\max } \\
{[\%]}\end{array}$ \\
\hline $\mathrm{A}$ & 1.50 & $0.0287(8)$ & 0.063 & $1.06(8)$ & $0.23(3)$ & $0.88(5)$ & $0.221(8)$ & $0.162(8)$ & $0.153(8)$ & $0.21(3)$ \\
$\mathrm{A}$ & 1.50 & $0.0387(4)$ & 0.085 & $2.30(4)$ & $0.21(1)$ & $2.0(2)$ & $0.215(3)$ & $0.130(3)$ & $0.28(2)$ & $0.20(3)$ \\
$\mathrm{A}$ & 1.50 & $0.0568(4)$ & 0.125 & $6.0(8)$ & $0.176(4)$ & $5.4(5)$ & $0.177(7)$ & $0.101(4)$ & $0.52(4)$ & $0.21(3)$ \\
$\mathrm{A}$ & 1.50 & $0.0765(3)$ & 0.168 & $16(1)$ & $0.20(1)$ & $16(1)$ & $0.211(5)$ & $0.076(5)$ & $0.93(8)$ & $0.28(4)$ \\
$\mathrm{A}$ & 1.50 & $0.1064(3)$ & 0.234 & $52(1)$ & $0.193(8)$ & $47(3)$ & $0.20(6)$ & $0.055(4)$ & $2.00(4)$ & $0.36(7)$ \\
& & & & & & & & & \\
B & 1.75 & $0.0263(1)$ & 0.058 & $0.48(5)$ & $0.22(7)$ & $0.35(4)$ & $0.23(2)$ & $0.27(8)$ & $0.10(2)$ & - \\
B & 1.75 & $0.0363(5)$ & 0.080 & $1.01(6)$ & $0.23(1)$ & $0.9(2)$ & $0.233(7)$ & $0.18(5)$ & $0.15(1)$ & $0.24(5)$ \\
B & 1.75 & $0.0552(1)$ & 0.121 & $2.9(4)$ & $0.21(3)$ & $2.7(3)$ & $0.214(2)$ & $0.13(1)$ & $0.32(4)$ & $0.29(2)$ \\
B & 1.75 & $0.0749(2)$ & 0.165 & $7.3(5)$ & $0.18(2)$ & $7.1(5)$ & $0.182(9)$ & $0.093(6)$ & $0.58(2)$ & $0.33(3)$ \\
B & 1.75 & $0.1032(2)$ & 0.227 & $23.7(7)$ & $0.19(1)$ & $22.6(6)$ & $0.185(7)$ & $0.072(4)$ & $1.14(3)$ & $0.51(5)$ \\
& & & & & & & & & \\
C & 2.00 & $0.0278(7)$ & 0.061 & $0.34(2)$ & $0.234(3)$ & $0.23(2)$ & $0.238(8)$ & $0.36(9)$ & $0.09(2)$ & - \\
C & 2.00 & $0.0383(3)$ & 0.084 & $0.71(7)$ & $0.21(2)$ & $0.57(3)$ & $0.22(1)$ & $0.16(1)$ & $0.11(2)$ & $0.23(6)$ \\
C & 2.00 & $0.0579(3)$ & 0.127 & $2.2(2)$ & $0.21(3)$ & $2.0(1)$ & $0.19(1)$ & $0.119(6)$ & $0.232(4)$ & $0.26(6)$ \\
C & 2.00 & $0.0783(5)$ & 0.172 & $4.9(4)$ & $0.20(1)$ & $4.9(5)$ & $0.195(7)$ & $0.10(1)$ & $0.40(1)$ & $0.34(6)$ \\
C & 2.00 & $0.1072(6)$ & 0.236 & $15.2(4)$ & $0.193(6)$ & $14.5(7)$ & $0.209(6)$ & $0.075(2)$ & $0.83(2)$ & $0.45(5)$ \\
\hline
\end{tabular}

We first discuss simulations of uniaxial deformation performed in the near-elastic range 
for the purpose of determining moduli. As an example of the data obtained from HMC, axial stress-strain curves from simulations of three type C aerogel models are shown in Figure 1. Data for gels of other densities and/or particle size is similar in apperance. The slopes of these data at zero strain are the Young's moduli of the materials. Significant thermal fluctuation is clearly visible, the absolute magnitude of which increases with the modulus, as expected based on statistical mechanical grounds. Note that the sinusoidal strain protocol described above spends more "time" at the maximum extensions than near zero strain, increasing the density of data in such regions. Moduli calculated from these simulations are collected in table 1. In order to confirm that the strain ranges used are appropriate (and within the elastic region) these simulations were also analyzed using only the "inner" $75 \%$ of the strain ranges explored (that is, $\pm 3.75 \%$ strain for the low-density gels and $\pm 1.875 \%$ strain for the high-density gels). No significant differences were observed in moduli extracted from the full and restricted data sets.

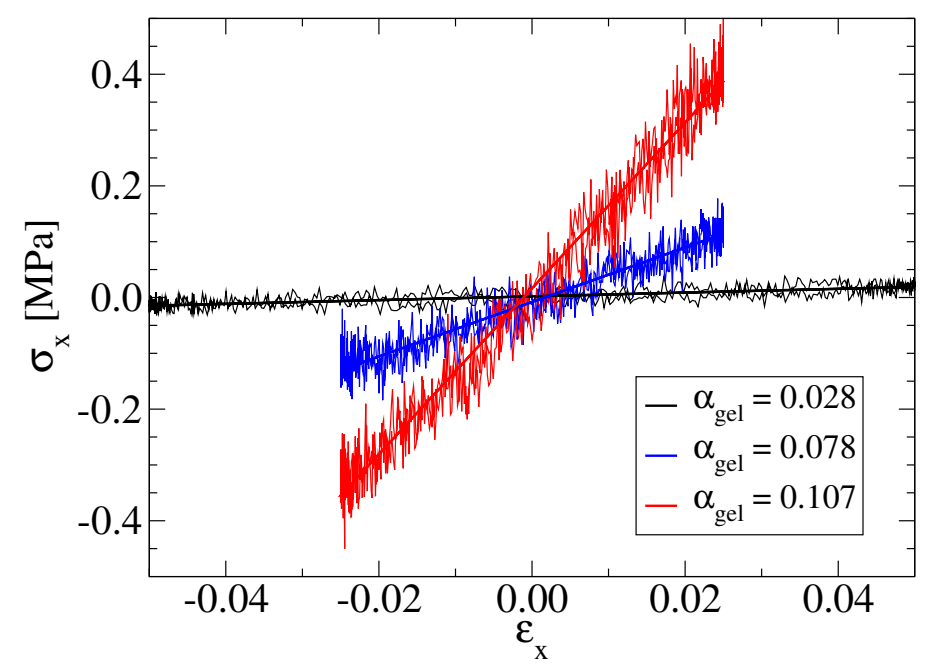

Figure 1: Representative stress-strain curves from HMC simulations, showing the behavior of three type $\mathrm{C}$ aerogels in the strain ranges used for the calculation of mechanical properties. Straight lines are linear regressions.

For the calculation of Young's modulus by MD we used the sinusoidal strain protocol discussed above with a fixed $\nu=0.20$. In denser aerogels $\left(\alpha_{\text {gel }} \geq 0.03\right)$, MD and HMC calculations of $Y$ agree to within estimated uncertainties, though HMC results are often 
slightly higher. For the lowest densities, the HMC data are systematically larger than MD results. This may be due to insufficient equilibration of one or the other methods; as discussed earlier, the lowest density gels have the longest time-scales of dynamics and relaxation. Some additional simulations of modestly longer and shorter length and/or strain range were performed, but did not resolve the discrepancy. We return to this point below.

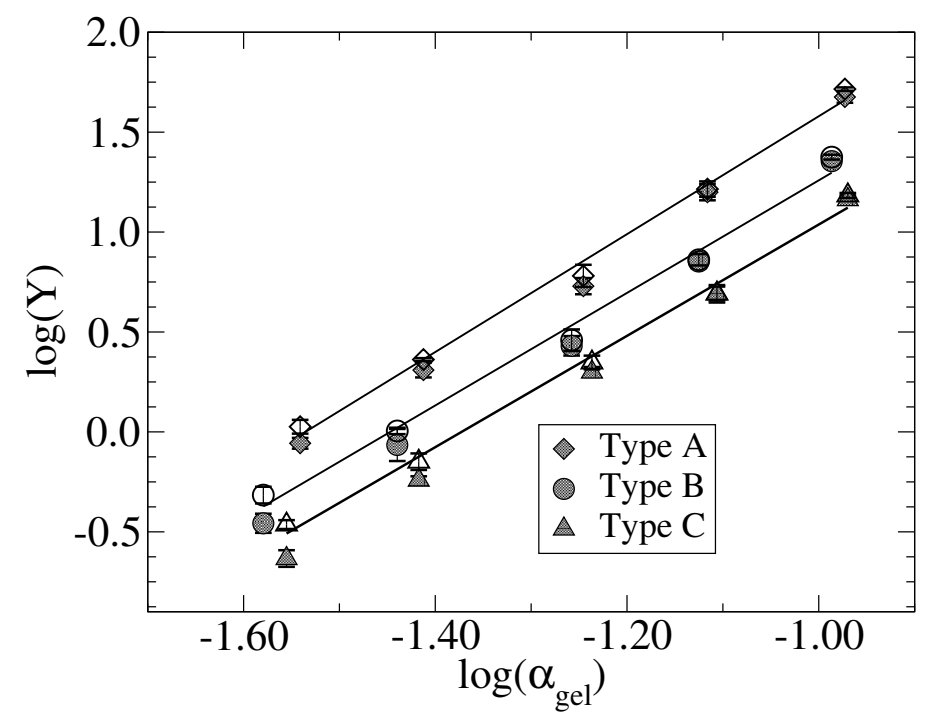

Figure 2: Dependence of $Y$ on $\alpha_{\text {gel }}$ for types A, B, and $\mathrm{C}$ aerogel models. Dark symbols are from MD calculations with $\nu=0.20$, while open symbols are HMC results; the lines are fits to the HMC results.

Regardless of the approach used, the Young's modulus increases with density according to a power-law with exponents of $2.9 \pm 0.1,2.8 \pm 0.2$, and $2.8 \pm 0.1$ for the types $\mathrm{A}, \mathrm{B}$, and $\mathrm{C}$ aerogels, respectively, as obtained from HMC simulations, or $3.0 \pm 0.1,3.0 \pm 0.1$, and $3.05 \pm 0.06$ if using $Y$ calculated by MD with assumed $\nu$. These data are shown in Figure 2. These exponents are comparable with those found experimentally, ${ }^{8,15,18,20,28}$ and agree within uncertainties with those found for the bulk modulus in previous work ${ }^{38}$.

Obtaining the Poisson ratio from MD simulations in which all the cell dimensions are controlled directly requires finding the value of $\nu$ that yields a constant transverse stress of zero as strain is applied. For this purpose we perform several independent simulations, with $\nu$ ranging 0.10 to 0.30 . If the imposed $\nu$ is too low, the gel cannot contract enough in the transverse direction and is under transverse tension, $(\delta \sigma / \delta \epsilon)_{\text {trans }}<0$. If the imposed 
$\nu$ is too high, then the gel is being compressed too much transverse to the applied strain, and $(\delta \sigma / \delta \epsilon)_{\text {trans }}>0$. The true Poisson ratio of the material can therefore be obtained by interpolating $(\delta \sigma / \delta \epsilon)_{\text {trans }}$ vs. $\nu$ data to find the intercept at which $(\delta \sigma / \delta \epsilon)_{\text {trans }}=0$. A quadratic function was used for this interpolation; representative data for type $\mathrm{C}$ aerogel models are shown in Figure 3.

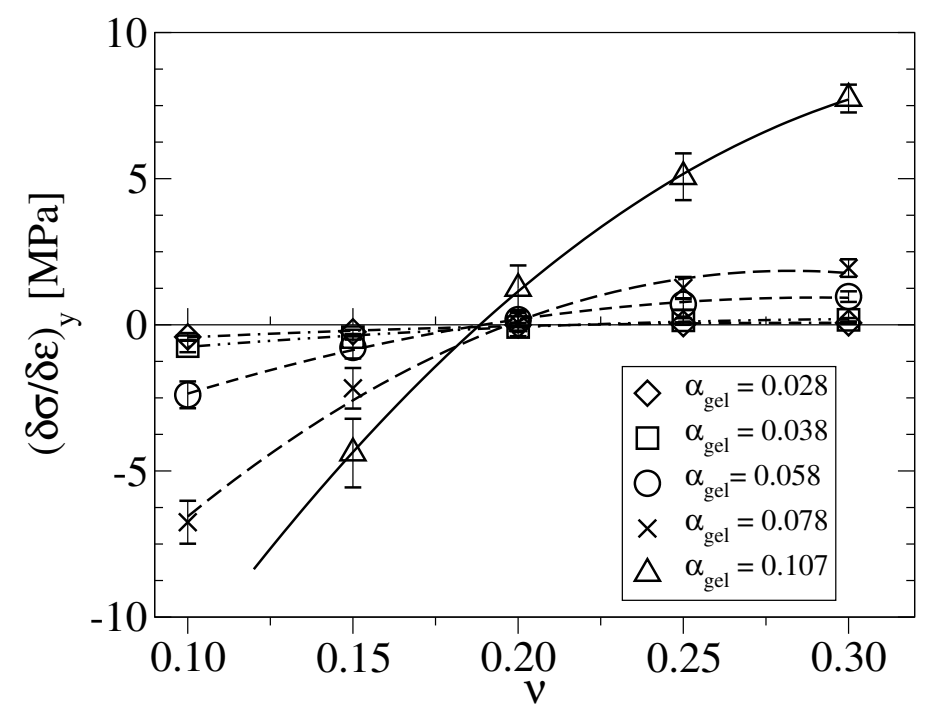

Figure 3: Estimating the Poisson ratio for type $\mathrm{C}$ aerogel models by means of MD simulations with given Poisson ratio. As the Poisson ratio is increased the perpendicular stress changes slope; using a smooth fit one may estimate the true Poisson ratio of the material as that which yields zero perpendicular stress.

Poisson ratios as determined for all the aerogel models by both HMC simulations (direct) and MD simulations (interpolation) are shown in Table 1. $\nu$ values in all cases vary between about 0.17 and 0.24 , with high-density gel values clustered around 0.20 or slightly below; these data are also plotted in Figure 4. Low density gels have somewhat higher $\nu$ than higher density gels. To the resolution of these simulations significant variation with density only occurs for $\alpha_{\text {gel }} \leq 0.05$ (approx. $0.1 \mathrm{~g} / \mathrm{cm}^{3}$ ), which is lower in density than most (though not all) experimentally studied aerogels.

From these simulations one can also extract axial stress-strain data, and obtain a $Y$-like quantity (since the transverse stress is not zero except at the true $\nu$, this is not exactly $Y$.) In all cases we find that as the imposed $\nu$ is increased, the slope of axial stress vs. strain 


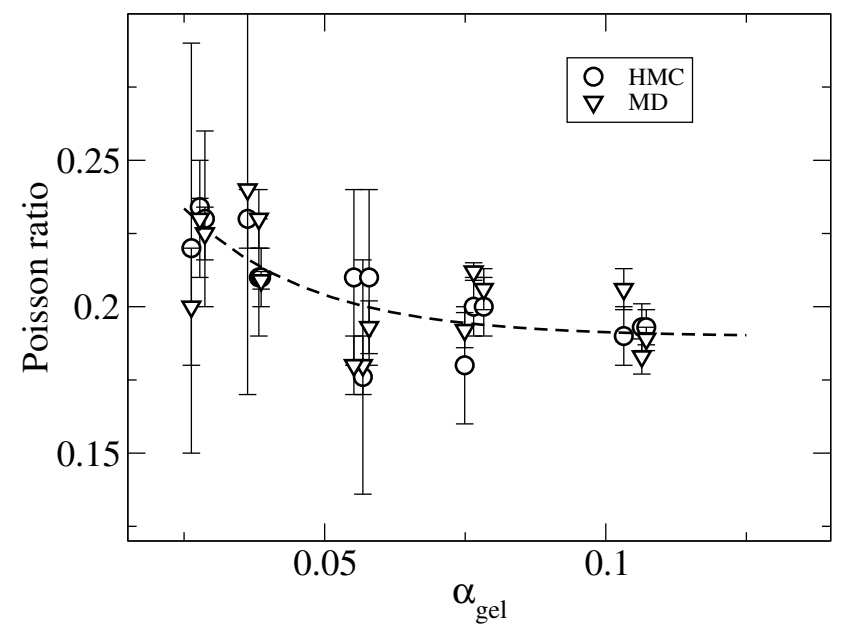

Figure 4: Dependence of Poisson ratios from both MD and HMC simulations on gel volume fraction. The curve is a guide to the eye.

decreases. Use of fixed $\nu=0.2$ for determination of Young's modulus by MD simulation therefore leads to systematically low $Y$ values for materials with $\nu<0.2$ and systematically high values for materials with $\nu>0.2$. This effect may explain the slightly low $Y_{M D}$ values at high density table 1 , but clearly not the low-density behavior; indeed, it suggests that the $Y_{M D}$ values (and therefore the $Y_{H M C}$ values) are systematically high.

As a test of isotropy, simulations of uniaxial deformation were performed along multiple cell axes in selected models, using both HMC and MD. The results, presented in Table 2, show that $Y$ and $\nu$ are independent of the direction of strain to within the resolution of these simulations, indicating that the simulation cells used are large enough to contain a representative samples of these materials.

Table 2: Evaluating isotropy of mechanical properties. $\alpha_{g e l}$ is the occupancy fraction of the gels, and $Y_{H M C}$ and $\nu_{H M C}$ are the the Young's modulus and Poisson ratio calculated by HMC simulations and the Poisson ratio calculated by MD simulations, with tension applied along the $\mathrm{x}, \mathrm{y}$, and $\mathrm{z}$ axis. Numbers in parentheses are the uncertainties in the last digit.

\begin{tabular}{cccccccccc}
\hline Model & $\alpha_{g e l}$ & $Y_{H M C}^{x}[\mathrm{MPa}]$ & $Y_{H M C}^{y}[\mathrm{MPa}]$ & $Y_{H M C}^{z}[\mathrm{MPa}]$ & $\nu_{H M C}^{x}$ & $\nu_{H M C}^{y}$ & $\nu_{H M C}^{z}$ & $\nu_{M D}^{y}$ & $\nu_{M D}^{z}$ \\
\hline A & $0.0287(8)$ & $1.06(8)$ & $0.97(8)$ & $0.99(6)$ & $0.23(3)$ & $0.24(3)$ & $0.22(2)$ & $0.225(9)$ & $0.221(8)$ \\
A & $0.1064(3)$ & $52(1)$ & $53(2)$ & $54(2)$ & $0.193(8)$ & $0.187(4)$ & $0.194(4)$ & $0.183(6)$ & $0.20(6)$ \\
& & & & & & & & \\
B & $0.02633(7)$ & $0.48(5)$ & $0.49(3)$ & $0.42(2)$ & $0.22(7)$ & $0.24(4)$ & $0.17(4)$ & $0.20(2)$ & $0.23(2)$ \\
B & $0.1032(2)$ & $23.7(7)$ & $23(2)$ & $23(1)$ & $0.19(1)$ & $0.190(6)$ & $0.18(2)$ & $0.206(7)$ & $0.185(7)$ \\
& & & & & & & & & \\
C & $0.0278(7)$ & $0.34(2)$ & $0.32(3)$ & $0.34(3)$ & $0.234(3)$ & $0.208(4)$ & $0.19(3)$ & $0.23(2)$ & $0.238(8)$ \\
C & $0.1072(6)$ & $15.2(4)$ & $15.2(5)$ & $14.3(3)$ & $0.193(6)$ & $0.194(4)$ & $0.197(9)$ & $0.189(4)$ & $0.209(6)$ \\
\hline
\end{tabular}


For homogeneous, isotropic, linear-elastic materials, the bulk modulus $K$ is related to $Y$ and $\nu$ by $K=Y /[3(1-2 \nu)]$. A test of this relationship for all the model aerogels is given in figure 5, using results for the bulk modulus obtained from compression/expansion $(c / e)$ simulations. ${ }^{38}$ Specifically, $K_{\text {hom }}$ is calculated from measured $Y$ and $\nu$ values, and the ratio $K_{c / e} / K_{\text {hom }}$ evaluated, which should be close to 1 . This is clearly true at high densities, but there is systematic deviation at low densities. The different deviations for HMC and MD-derived data seems likely related to the low-density disagreement between $Y_{H M C}$ and $Y_{M D}$ discussed above. Overestimation of $K_{\text {hom }}$ could be due either to overestimation of $Y$ or overestimation of $\nu$. The $\nu$ data do not vary sufficiently to explain the observed trend, so this data suggests, again, that both simulation techniques are yielding too-high values for $Y$ at the lowest densities. Note that these calculations are performed using the average $Y$, $\nu$, etc., data for each group of three model realizations, rather than independently for each specific model instance, which may also contribute to the deviation from expected behavior.

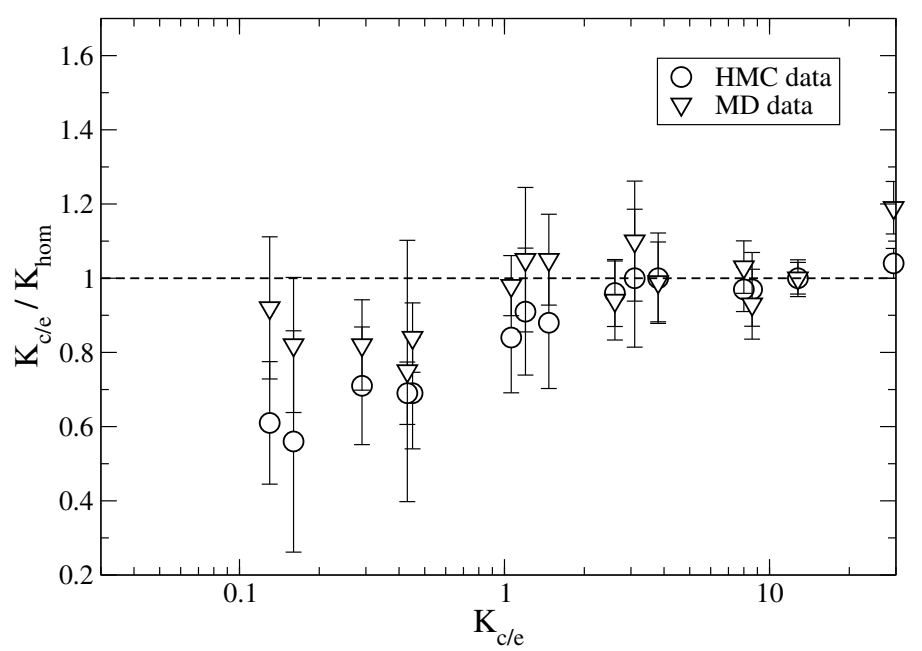

Figure 5: Bulk modulus from compression-expansion simulations, $K_{c / e}$, compared with that calculated from $Y$ and $\nu$ assuming a homogeneous isotropic medium. Data are shown for both HMC and MD results. Uncertainties are calculated assuming independence of uncertainties in each constituent quantity. 


\subsection{Behavior under large deformations}

We now turn to tension and compression beyond the elastic range. Figure 6 shows snapshots of a type $\mathrm{C}$ aerogel model of $\alpha_{\text {gel }}=0.028$ (97\% porosity, the highest studied) at (a) $40 \%$ compressive strain, (b) uncompressed, and (c) $20 \%$ tensile strain. While the uncompressed system appears isotropic, the texture of the compressed material is significantly different. In the gel under tension, (c), "strands" of gel network aligned in the direction of strain are visible. The dense clusters of gel particles found in the central image are easily identified in the compressed and extended structures at left and right, though often rotated. This suggests that strain is accomodated by the more tenuous linkages between the clusters, and therefore that most of the local structure is unperturbed even at these large deformations.
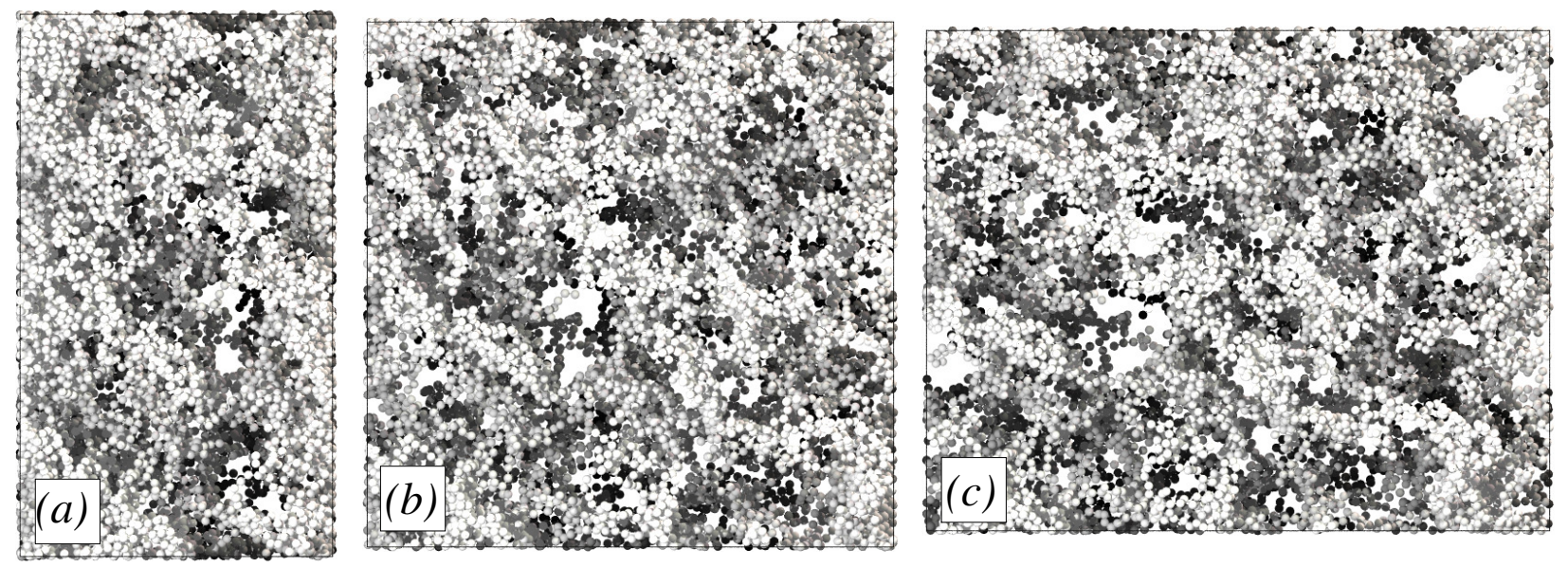

Figure 6: Snapshots of a type C aerogel model with $\alpha_{\text {gel }}=0.028$ (a) after $40 \%$ compression strain, (b) uncompressed, and (c) after 20\% tension strain is applied. Particles are colored by depth, with those at the front of the simulation cell in white, and those at the back in dark grey.

Stress-strain curves for type C aerogel models under high compression and tension are shown in Figure 7; very similar behavior was observed for type A and B models. Under compression, the two lowest-density aerogels exhibit elastic behavior over the entire range studied, while denser gels have much smaller elastic ranges and display abrupt transitions to plastic behavior. At $\alpha_{g e l}=0.058$, the model begins to display plastic behavior above about $30 \%$ compression, and for $\alpha_{\text {gel }}=0.078$ the elastic-to-plastic crossover occurs at about $10 \%$ 

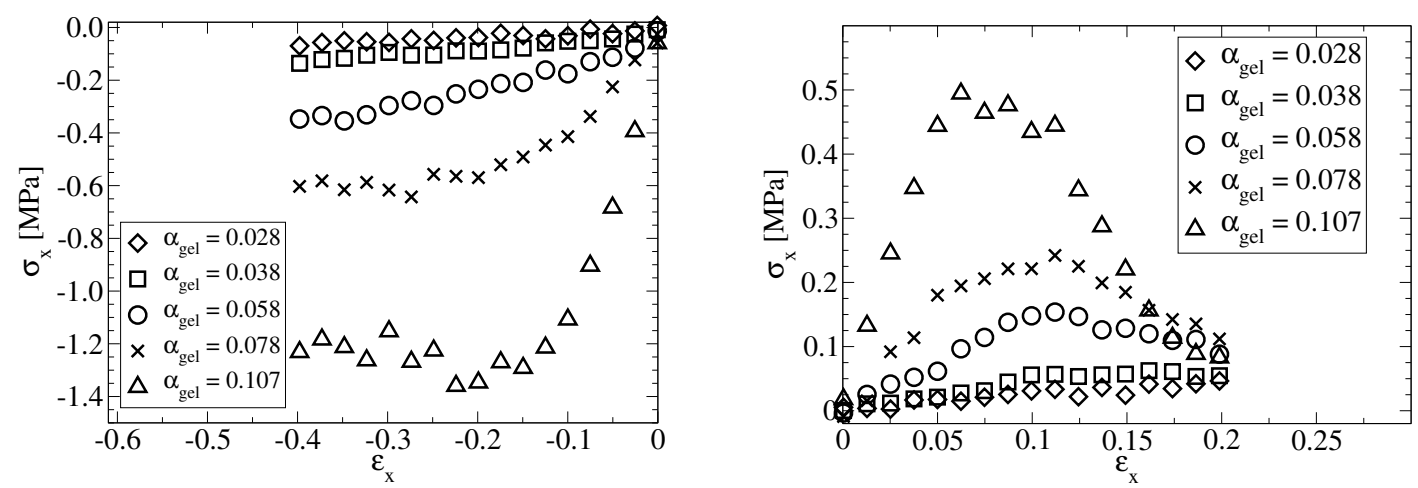

Figure 7: Stress-strain curves for type C aerogel models under large compressions (left) and tensions (right).

compression. In the densest gel, at $\alpha_{\text {gel }}=0.107$, the elastic regime ends between $5 \%$ and $10 \%$ compression, and at compressions greater than $20 \%$ the magnitude of the axial stress drops, suggesting that significant damage has been done to the gel structure.

Under tension, the lowest-density gel displays a nearly elastic response over the entire range studied. For $\alpha_{\text {gel }} \geq 0.038$ an elastic-to-plastic crossover occurs below $10 \%$ elongation. The three highest-density materials each show a well-defined maximum in the axial stress, the position of which moves to lower strain as the density is increased. This is consistent with the finding from compressive simulations that the low density models have large elastic ranges while the high density models have much smaller ones, and undergo brittle failure. We note that similar qualitative behavior is found in experimental work on polyethoxydisiloxane aerogels, ${ }^{18}$ where materials with densities above $0.2 \mathrm{~g} / \mathrm{cm}^{3}$ were found to be very brittle while those of low density $\left(\leq 0.1 \mathrm{~g} / \mathrm{cm}^{3}\right)$ are ductile.

Transverse vs. axial strain data are shown in Figure 8, and point to important qualitative differences between high and low density aerogels. Models of all densities display negative curvature in the range $-0.20<\epsilon_{x}<-0.05$, just outside of the elastic region, which indicates a reduction in the effective Poisson ratio. However, for high density models the curvature becomes positive for larger compressions, and $\nu$ edges towards 0.5 , the incompressible limit. These materials therefore still possess the ability to transfer stress between axial and transverse directions, though have sustained significant damage by this point. Low-density 


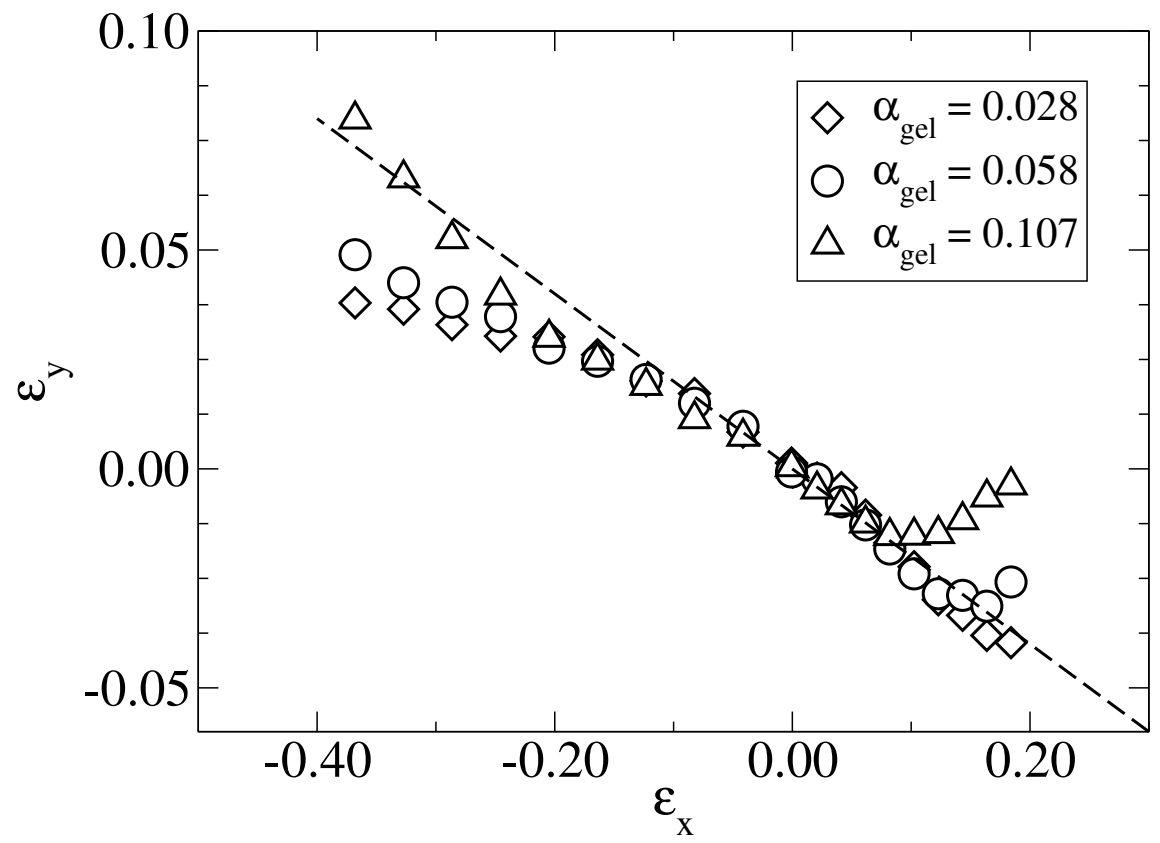

Figure 8: Transverse $(y)$ strain vs. axial $(x)$ strain in type $\mathrm{C}$ aerogels with $\alpha_{\text {gel }}=0.028$, 0.058, and 0.107, from large-extension HMC simulations. A linear fit in the elastic region gives the Poisson ratio for these materials. The dotted line has a slope of -0.20.

aerogels, however, show only negative curvature under compression, and the $\alpha_{\text {gel }}=0.058$ data appears near to an inflection point at $40 \%$ compression.

Under tension, the data in Figure 8 again display negative curvature for small deformations $\left(\epsilon_{x}<0.10\right)$, indicating a reduction of effective $\nu$. At higher tensions the data inflect and the curvature becomes positive, indicating a negative effective $\nu$ (auxetic behavior); this effect is quite strong for the highest density aerogel. Interestingly, the minima in these curves (where $\nu=0$ ) occur at strains somewhat higher than do the maxima in the tensile stress, so that damage to the material is likely involved. This behavior is not observed for the lowest-density gel studied, though it is possible that it occurs at larger strains that were studied; the change in curvature at the largest strains suggests this may be so.

We note that the coarse-grained nature of the model limits its use in simulations of large compression strain. This simulation approach is unsuitable for work at conditions where deformation or coalescence of the primary gel particles is expected. Under extreme compression the coarse-grained model will favor a close-packing of the primary particles, while 
a real aerogel will become first a mesoporous xerogel-like structure and finally a nonporous solid. The compressive simulations therefore cannot be continued too far without unphysical results. Packing between incompressible particles may contribute to the upturn in perpendicular strain at high compressions displayed by the densest gel in Figure 8. Tensile deformation simulations are unaffected by this issue, however. The ability to perform simulations under extreme tension is a useful feature of this model; in experimental work tensile deformation of aerogels is very difficult to study.
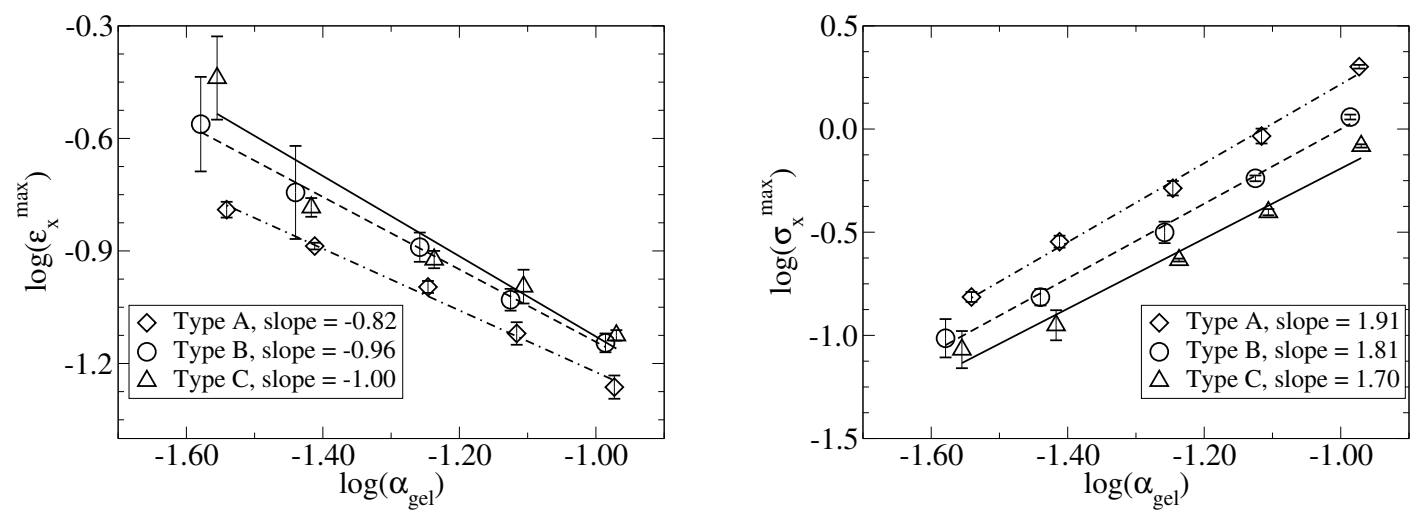

Figure 9: Dependence of the strain at maximum tensile stress on $\alpha_{\text {gel }}$ (left), and dependence of the maximum tensile stress on $\alpha_{\text {gel }}$ (right), with power-law exponents given in the legends.

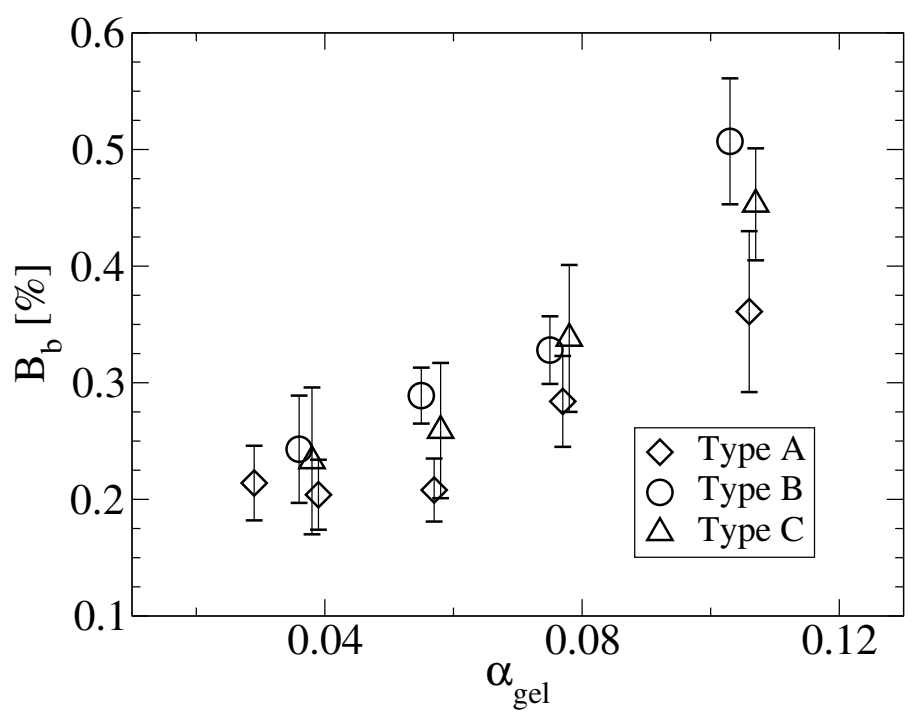

Figure 10: Percentage of bonds broken at the point of maximum tensile stress vs. $\alpha_{g e l}$, for types A, B, and C aerogel models.

Collected estimates of the maximum tensile stress $\sigma_{\max }$ and corresponding strain $\epsilon_{\max }$ are 
shown in Figure 9, with data also given in Table 1. Although these are rather noisy quantities to measure, both display clear power-law dependence on the gel density; the maximum tensile stress (that is, the tensile strength) exponents are comparable but somewhat lower than the value of 2.3 value obtained by Wong et al. ${ }^{18}$

As a microscopic metric of the damage sustained during tensile simulations, we calculated the fraction of bonds broken at the strain of maximum stress, shown in Figure 10. This quantity clearly increases with aerogel density; the lowest density materials only exhibit around $0.2 \%$ bond breakage at maximum stress, while in the higher density materials this reaches around $0.5 \%$. Since the number of bonds is nearly linear with the number of particles ${ }^{38}$, the actual number of bonds broken at this point increases rather more rapidly. The data in Figure 10 exhibit some curvature, but it is difficult to quantify.

In all cases the fraction of bonds broken at maximum tensile stress is well under $1 \%$. This is interesting given that previous studies suggest that as few as $10 \%$ of bonds may carry most of the load in these materials. ${ }^{34}$ If this is the case, then breakage of $0.5 \%$ of bonds should perhaps be interpreted as a $5 \%$ disruption of the load-bearing network, which seems more consistent with the large changes observed in the stress-strain and strain-strain data.

We have also considered bond breakage upon compression (data not shown). In models with $\alpha_{g e l} \geq 0.078$, maximum compressive stresses are also identifiable (see figure 7 ), and at those points between 1 and $2 \%$ of the bonds are broken, considerably more than the number broken at maximum tensile strain. 


\section{Conclusions}

Uniaxial deformations and associated mechanical properties of model aerogels were studied using both molecular dynamics and hybrid Monte Carlo methods. Measurement of moduli in these materials is challenging from a simulation standpoint. Aerogels are "soft" in comparison with most solids, but "hard" in the categorization of materials into "hard" and "soft" matter, since the chemical bonds between particles are much stronger than $k_{B} T$.

Young's moduli from HMC and MD simulations agreed well for all but the lowest-density model aerogels, which are both the softest and the most difficult to simulate as they display the longest-wavelength fluctuations. The moduli of the model materials displays a power-law dependence on aerogel density with an exponent of approximately 3.0, nearly independent of constituent particle size. This exponent is within the range reported in the literature ${ }^{8,15,18,20,28}$ and very similar to that previously found for the bulk modulus in the same models. ${ }^{38}$ Measurements of modulus and Poisson's ratio along different axes indicated that these properties are isotropic to within the uncertainty of the data, though there is rather more anisotropy for the low-density materials than for the high density ones. Higherdensity models have moduli consistent with homogeneous elastic medium theory, but there are deviations observed at very low densities consistent with overestimation of $Y$ by both methods, suggesting that considerably longer simulations are required to access the true moduli of very low density materials.

Our models yield Poisson ratios between 0.17 and 0.23 , with higher-density gels clustered around 0.20, in good agreement with values measured (and assumed) in the literature. ${ }^{7,9,21,24-26}$ This agreement is rather better than that obtained in previous simulation studies of aerogels using finite-element approaches. ${ }^{39} \mathrm{~A}$ weak dependence of Poisson ratio on density was observed, with increased $\nu$ at the lowest densities. Experimental measurements of $\nu$ with density at very low densities have not appeared in the literature to date. Computer graphics visualizations suggest that at very low densities aerogels are composed of tenuous strands of particles connecting larger clusters. This picture is consistent with the increase 
in $\nu$, as such strands, though very weak, still effectively couple axial and transverse stresses.

We also performed simulations of uniaxial compressions and tensions of large magnitude. Low density aerogels are found to have a much larger elastic range, with properties changing only gradually throughout. High density aerogels fail abruptly under both tension and compression and exhibit significant changes in Poisson ratio under compression, suggesting brittle behavior. A similar ductile-to-brittle transition has been observed in experimental work. These simulations also displayed power-law variation of tensile strength in reasonable accord with experiments, and power-law variation in the strain at maximum stress. The models displayed auxetic behavior at large tensile strain, which was unexpected. Analysis of the model structures at the point of maximum tensile stress indicated that very few bonds were broken at that point, supporting the hypothesis that much of the gel network does not contribute to the mechanical strength of these materials. ${ }^{39}$

To summarize, we have performed detailed mechanical characterization of a range of aerogel models, finding that they display behavior both quantitatively and qualitatively in agreement with experimental materials. Most experimental work is performed on materials occupying to the higher end of the density range investigated here, so this modeling approach should be useful in better understanding real materials of practical interest. It appears that further investigation of the behavior of aerogels of volume fraction below 0.03 will require significantly longer simulations, and possibly larger simulation cells as well. Nonetheless, our results suggest that coarse-grained simulation will be a reliable way to probe structural evolution and failure modes in aerogels under different kinds of mechanical strain, and that simulations of this type could even be used in the design of aerogel-based hybrids and nanomaterials for improved mechanical performance. 


\section{Acknowledgments}

We acknowledge financial support from the National Science Foundation (CHE-0626008 and CHE-0718861), and the ARO (W911NF-07-1-0253). Some of these calculations were performed on NSF XSEDE resources (allocation MCA08X001).

\section{References}

(1) Pierre, A. C. Aerogels Handbook; Advances in Sol-Gel Derived Materials and Technologies; Springer: New York, 2011; Chapter 1, pp 3-18.

(2) Gurav, J. L.; Jung, I.; Park, H.; Kang, E. S.; Nadargi, D. Y. Silica Aerogel: Synthesis and Applications. J. of Nanomaterials 2010, 2010, 1-11.

(3) Aegerter, M. A., Leventis, N., Koebel, M. M., Eds. Aerogel Handbook; Advances in Sol-Gel Derived Materials and Technologies; Springer: New York, 2011.

(4) Brinker, C. J.; Scherer, G. W. Sol-Gel Science; Acad. Press: San Diego, 1990.

(5) Pierre, A. C.; Rigacci, A. Aerogels Handbook; Advances in Sol-Gel Derived Materials and Technologies; Springer: New York, 2011; Chapter 2, pp 21-46.

(6) Dorcheh, S.; Abbasi, M. Silica aerogel; synthesis, properties and characterization. J. Mater. Process. Tech. 2008, 199, 10-26.

(7) Scherer, G. W.; Smith, D. M.; Qiu, X.; Anderson, J. M. Compression of Aerogels. J. Non-Cryst. Solids 1995, 186, 316-320.

(8) Woignier, T.; Phalippou, J. Scaling law variation of the mechanical properties of silica aerogels. Revue de Physique Appliquée 1989, 24, 179-184.

(9) Woignier, T.; Reynes, J.; Hafidi Alaoui, A.; Beurroies, I.; Phalippou, J. Different kinds of structure in aerogels: relationships with the mechanical properties. J. NonCryst. Solids 1998, 241, 45-52. 
(10) Álvarez Arenas, T. E. G.; de Espinosa, F. R. M. Viscoelasticity of silica aerogels at ultrasonic frequencies. Applied Physics Letters 2002, 81, 1198-1200.

(11) Kucheyev, S. O.; Hamza, A. V.; Satcher, Jr., J. H.; Worsley, M. A. Depth-sensing indenstation of low-density brittle nanoporous solids. Acta Materialia 2009, 57, 34723480 .

(12) Nikel, O.; Anderson, A. M.; Carroll, M. K.; Keat, W. D. Effect of uni-axial loading on the nanostructure of silica aerogels. J. Non-Cryst. Solids 2011, 357, 3176-3183.

(13) Fricke, J.; Emmerling, A. Aerogels. J. Am. Ceram. Soc 1992, 75, 2027-2036.

(14) Reichenauer, G.; Scherer, G. W. Nitrogen adsorption in compliant materials. J. NonCryst. Solids 2000, 27r, 162-172.

(15) Woignier, T.; Phalippou, J.; Sempere, R.; Pelous, J. Analysis of the elastic behaviour of silica aerogels taken as a percolating system. J. Phys. France 1988, 49, 289-293.

(16) Gross, J.; Scherer, G. W. Dynamic pressurization: novel method for measuring fluid permeability. J. Non-Crystalline Solids 2003, 325, 34-47.

(17) Alaoui, A. H.; Woignier, T.; Scherer, G. W.; Phalippou, J. Comparison between flexural and uniaxial compression tests to measure the elastic modulus of silica aerogel. J. NonCrystalline Solids 2008, 354, 4556-4561.

(18) Wong, J. C. H.; Kaymak, H.; Brunner, S.; Koebel, M. M. Mechanical Properties of Monolithic Silica Aerogels made from Polyethoxydisiloxanes. Microporous and Mesoporous Materials 2013,

(19) Stark, R. W.; Drobek, T.; Weth, M.; Fricke, J.; Heckl, W. M. Determination of elastic properties of single aerogel powder partivles with the AFM. Ultramicroscopy 1998, 75, $161-169$. 
(20) Moner-Girona, M.; Roig, A.; Molins, E. Micromechanical properties of silica aerogels. Applied Physics Letters 1999, 75, 653-655.

(21) Gross, J.; Reichenauer, G.; Fricke, J. Mechanical properties of $\mathrm{SiO}_{2}$ aerogels. J. Phys. D: Appl. Phys. 1988, 21, 1447-1451.

(22) Pollanen, J.; Shirer, K. R.; Blinstein, S.; Davis, J. P.; Choi, H.; Lippman, T. M.; Halperin, W. P.; Lurio, L. B. Globally anisotropic high porosity silica aerogels. J. NonCrystalline Solids 2008, 354, 4668-4674.

(23) Bhupathi, P.; Jaworski, L.; Hwang, J.; Tanner, D. B.; Obukov, S.; Lee, Y.; Mulders, N. Optical birefringence in uniaxially compressed aerogels. New Journal of Physics 2010, 12, 103016-103036.

(24) Forest, L.; Gibiat, V.; Woignier, T. Biot's theory of acoustic propagation in porous media applied to aerogels and alcogels. J. Non-Crystalline Solids 1998, 225, 287-292.

(25) Kucuk, Y. Determination of temperature effect on mechanical properties of templated mesoporous silica aerogels by nanoindentation method. Int. J. Phys. Sciences 2011, 6, $5369-5375$.

(26) Kucheyev, S. O.; Stadermann, M.; Shin, S. J.; Satcher, Jr., J. H.; Gammon, S. A.; Letts, S. A.; van Buuren, T.; Hamza, A. V. Super-Compressibility of Ultralow-Density Nanoporous Silica. Adv. Mater. 2012, 24, 776-780.

(27) Reichenauer, G.; Scherer, G. W. Extracting the pore size distribution of compliant materials from nitrogen adsorption. Colloids and Surfaces 2001, 187-188, 41-50.

(28) Phalippou, J.; Despetis, F.; Calas, S.; Faivre, A.; Dieudonné, P.; Sempéré, R.; Woignier, T. Comparison between sintered and compressed aerogels. Optical Materials 2004, 26, 167-172. 
(29) Miner, M. R.; Hosticka, B.; Norris, P. M. The effects of ambient humidity on the mechanical properties and surface chemistry of hygroscopic silica aerogel. J. NonCryst. Solids 2004, 350, 285-289.

(30) de la Rosa-Fox, N.; Morales-Flórez, V.; Toledo-Fernández, J. A.; Piñero, M.; MendozaSerna, R.; Esquivias, L. Nanoindentation on hybrid organic/inorganic silica aerogels. J. of the European Ceramic Society 2007, 27, 3311-3316.

(31) Meador, M. A. B.; Capadona, L. A.; McCorkle, L.; Papadopoulos, D. S.; Leventis, N. Structure-Property Relationships in Porous 3D Nanostructures as a Function of Preparation Conditions: Isocyanate Cross-Linked Silica Aerogels. Chem. Mater. 2007, 19, $2247-2260$.

(32) Martín, L.; Ossó, J. O.; Ricart, S.; Roig, A.; García, O.; Sastre, R. Organo-modified siliica aerogels and implications for material hydrophobicity and mechanical properties. J. Mater. Chem. 2008, 18, 207-213.

(33) Obrey, K. A. D.; Wilson, K. V.; Loy, D. A. Enhancing mechanical properties of silica aerogels. J. Non-Crystalline Solids 2011, 35\%, 3435-3441.

(34) Ma, H.-S.; Roberts, A. P.; Prévost, J.-H.; Jullien, R.; Scherer, G. W. Mechanical structure-property relationship of aerogels. J. Non-Crystalline Solids 2000, 27r, 127141.

(35) Ma, H.-S.; Prévost, J.-H.; Jullien, R.; Scherer, G. W. Computer simulation of mechanical structure-property relationship of aerogels. J. Non-Cryst. Solids 2001, 285, $216-221$.

(36) Lu, H.; Fu, B.; Daphalapurkar, N.; Hanan, J. Simulation of the evolution of the nanostructure of crosslinked silica-aerogels under compression. Polymer Preprints 2008, 49, $564-565$. 
(37) Fu, B.; Luo, H.; Wang, F.; Churu, G.; Chu, K. T.; Hanan, J. C.; Sotiriou-Leventis, C.; Leventis, N.; Lu, H. Simulation of the microstructural evolution of a polymer crosslinked templated silica aerogel under high-strain-rate compression. J. Non-Cryst. Solids 2011, 357, 2063-2074.

(38) Ferreiro-Rangel, C. A.; Gelb, L. D. Investigation of the bulk modulus of silica aerogel using molecular dynamics simulations of a coarse-grained model. J. Phys. Chem. B 2013, 117, 7095-7105.

(39) Ma, H.-S.; Prévost, J.-H.; Scherer, G. W. Elasticity of DLCA model gels with loops. Int. J. of Solids and Structures 2002, 39, 4605-4614.

(40) Barbero, E. J.; Campo, F. A. Sol-gel simulation-II: Mechanical response. J. NonCryst. Solids 2012, 358, 728-734.

(41) Gelb, L. D. Simulating Silica Aerogels with a Coarse-Grained Flexible Model and Langevin Dynamics. J. Phys. Chem. C 2007, 111, 15792-15802.

(42) Bijsterbosch, B. H.; Bos, M. T. A.; Dickinson, E.; van Opheusden, J. H. J.; Walstra, P. Brownian Dynamics Simulation of Particle Gel Formation: From Argon to Yoghurt. Faraday Discuss. 1995, 101, 51-64.

(43) Whittle, M.; Dickinson, E. Stress overshoot in a model particle gel. J. Chem. Phys. 1997, 107, 10191-10200.

(44) Rzepiela, A. A.; van Opheusden, J. H. J.; van Vliet, T. Brownian Dynamics Simulation of Aggregation Kinetics of Hard Spheres with Flexible Bonds. J. Colloid. Int. Sci. 2001, $244,43-50$.

(45) d'Arjuzon, R. J. M.; Frith, W.; Melrose, J. R. Brownian dynamics simulations of aging colloidal gels. Phys. Rev. E 2003, 67, 061404. 
(46) Gillespie, D. T. A General Method for Numerically Simulating the Stochastic Time Evolution of Coupled Chemical Reactions. J. Comp. Phys. 1976, 22, 403-434.

(47) Parrinello, M.; Rahman, A. Polymorphic transitions in single crystals: A new molecular dynamics method. J. Appl. Phys. 1981, 52, 7182-7190.

(48) Duane, S.; Kennedy, A. D.; Pendleton, B. J.; Roweth, D. Hybrid Monte Carlo. Phys. Lett. B 1987, 195, 216-222.

(49) Mehlig, B.; Heermann, D. W.; Forrest, B. M. Hybrid Monte Carlo method for condensed-matter systems. Phys. Rev. B 1992, 45, 679-685.

(50) Brass, A.; Pendleton, B. J.; Chen, Y.; Robson, B. Hybrid Monte Carlo Simulations Theory and Initial Comparison with Molecular Dynamics. Biopolymers 1993, 33, 13071315.

(51) Clamp, M. E.; Baker, P. G.; Stirling, C. J.; Brass, A. Hybrid Monte Carlo: An efficient algorithm for condensed matter simulation. J. Comp. Chem. 1994, 15, 838-846.

(52) Beskos, A.; Pillai, N. S.; Roberts, G. O.; Sanz-Serna, J. M.; Stuart, A. M. Optimal tuning of the Hybrid Monte-Carlo algorithm. Arxiv:1001.4460 2010,

(53) Allen, M. P.; Tildesley, D. J. Computer Simulation of Liquids; Clarendon Press: Oxford, 1987.

(54) Lubarda, V. A. On thermodynamic potentials in linear thermoelasticity. Int. J. of Solids and Structures 2004, 41, 7377-7398. 